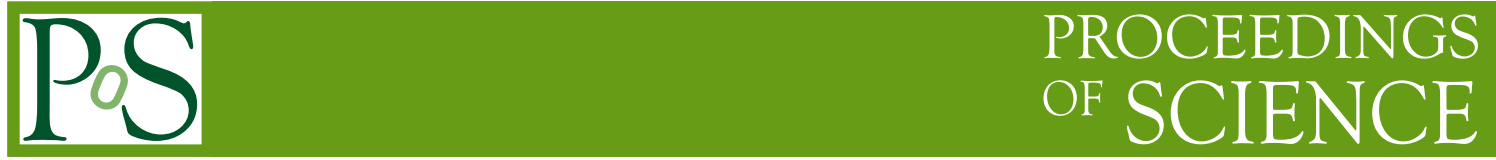

\title{
Evidence for an anomalous like-sign dimuon charge asymmetry
}

\section{Bruce Hoeneisen ${ }^{*}$}

Universidad San Francisco de Quito

E-mail: bruce1@fnal.gov

We measure the charge asymmetry $A \equiv\left(N^{++}-N^{--}\right) /\left(N^{++}+N^{--}\right)$of like-sign dimuon events in $6.1 \mathrm{fb}^{-1}$ of $p \bar{p}$ collisions recorded with the D0 detector at a center-of-mass energy $\sqrt{s}=$ $1.96 \mathrm{TeV}$ at the Fermilab Tevatron collider. From $A$ we extract the like-sign dimuon charge asymmetry in semileptonic $b$-hadron decays: $A_{\mathrm{sl}}^{b}=-0.00957 \pm 0.00251$ (stat) \pm 0.00146 (sys). It differs by 3.2 standard deviations from the standard model prediction $A_{\mathrm{sl}}^{b}(\mathrm{SM})=\left(-2.3_{-0.6}^{+0.5}\right) \times 10^{-4}$, and provides the first evidence of anomalous $C P$ violation in the mixing of neutral $B$ mesons.

35th International Conference of High Energy Physics

July 22-28, 2010

Paris, France

\footnotetext{
* Speaker.

${ }^{\dagger}$ Representing the DØ Collaboration
} 
A detailed description of the analysis [1] and a letter [2] have been recently published. This conference note is a short version of [2].

Studies of particle production and decay under the reversal of discrete symmetries (charge conjugation (C), parity (P) and time reversal) have yielded considerable insight into the structure of theories that describe high energy phenomena. The violation of these discrete symmetries has only been observed in the weak interaction. Of particular interest is the observation of $C P$ violation [3], a phenomenon well established in the $K^{0}$ and $B_{d}^{0}$ systems, but not in the $B_{s}^{0}$ system where the effects of $C P$ violation are expected to be small in the standard model (SM) [4]. A review of the experimental results and of the theoretical framework for describing $C P$ violation in the mixing of $B_{q}^{0} \leftrightarrow \bar{B}_{q}^{0}(q=d, s)$ mesons [5] can be found in Ref. [6]. At the Fermilab Tevatron collider, $b$ quarks are produced mainly in $b \bar{b}$ pairs. In like-sign dimuon events, one muon can arise from direct semileptonic decay, e.g., $b \rightarrow \mu^{-} X$, of a $\bar{B}_{q}^{0}$ or $B^{-}$meson, and the other from a $B_{q}^{0} \leftrightarrow \bar{B}_{q}^{0}$ oscillation followed, in this example, by a semileptonic decay of the $\bar{B}_{q}^{0}$ meson, $B_{q}^{0} \rightarrow \bar{B}_{q}^{0} \rightarrow \mu^{-} X$. Extensions of the SM containing additional contributions to the Feynman "box" diagrams responsible for $B_{q}^{0}$ mixing may enhance $C P$ violation in mixing $[7,8,9,10,11]$. The violation of $C P$ symmetry is a necessary condition for baryogenesis, the process thought to be responsible for the matterantimatter asymmetry of the universe [12]. However, the observed level of $C P$ violation in the $K^{0}$ and $B_{d}^{0}$ systems is not sufficient to accommodate this asymmetry, suggesting the presence of additional sources of $C P$ violation beyond the SM [13].

The data, corresponding to an integrated luminosity of $6.1 \mathrm{fb}^{-1}$, were recorded with the D0 detector [14] at the Fermilab Tevatron proton-antiproton $(p \bar{p})$ collider, operating at a center-of-mass energy of $1.96 \mathrm{TeV}$. The D0 experiment is well suited to the investigation of the small effects of $C P$ violation because the periodic reversal of the $\mathrm{D} 0$ solenoid and toroid magnetic field polarities results in a cancellation of most detector-related charge asymmetries. In addition, the $p \bar{p}$ initial state is $C P$-invariant, and the high center-of-mass energy provides access to mass states beyond the reach of the $B$-factories running at $\sqrt{s}=M(\Upsilon(4 S))<2 M\left(B_{s}\right)$.

The like-sign dimuon charge asymmetry $A$ is defined as

$$
A \equiv \frac{N^{++}-N^{--}}{N^{++}+N^{--}}
$$

where $N^{++}$and $N^{--}$represent the number of events in which the two muons with the highest $p_{T}$, defined as the momentum component transverse to the beam axis, satisfying the kinematic selections, have the same positive or negative charges. After removing contributions from background and from remaining detector effects, any residual asymmetry is assumed to arise solely from the mixing of $B_{q}^{0}(q=d, s)$ mesons (via $B_{q}^{0} \leftrightarrow \bar{B}_{q}^{0}$ oscillations) that later decay semileptonically. This corrected asymmetry $A_{\mathrm{sl}}^{b}$ is defined as

$$
A_{\mathrm{sl}}^{b} \equiv \frac{N_{b}^{++}-N_{b}^{--}}{N_{b}^{++}+N_{b}^{--}}
$$

where $N_{b}^{++}$and $N_{b}^{--}$represent the number of events containing two $b$-hadrons decaying semileptonically into two positive or two negative muons, respectively. Assuming CPT invariance, each neutral $B_{q}^{0}$ meson contributes a term to this asymmetry

$$
A_{\mathrm{sl}}^{b}=\beta_{d} a_{\mathrm{sl}}^{d}+\beta_{s} a_{\mathrm{sl}}^{s},
$$


with

$$
a_{\mathrm{sl}}^{q}=\frac{\Delta \Gamma_{q}}{\Delta M_{q}} \tan \phi_{q}
$$

where $\phi_{q}$ is the $C P$-violating phase, and $\Delta M_{q}$ and $\Delta \Gamma_{q}$ are the mass and width differences between the eigenstates of the propagation matrices of the neutral $B_{q}^{0}$ mesons. The values of $\beta_{d}=0.506 \pm$ 0.043 and $\beta_{s}=0.494 \pm 0.043$ are taken from previous measurements [6]. The SM predicts [4, 1]:

$$
A_{\mathrm{sl}}^{b}(\mathrm{SM})=\left(-2.3_{-0.6}^{+0.5}\right) \times 10^{-4}
$$

and an experimental result significantly different from this expectation would therefore be indicative of the presence of physics beyond the SM.

The main background for these measurements arises from events with at least one muon from kaon or pion decay or from the sequential decay of $b$ quarks, $\bar{b} \rightarrow \bar{c} \rightarrow \mu^{-} X$. The most important background asymmetry arises from the fact that $K^{+}$and $K^{-}$mesons interact differently with the material of the detector, and thus their decay rates into positive and negative muons are not identical.

The asymmetry $A_{\mathrm{sl}}^{b}$ can also be obtained from the measurement of the charge asymmetry $a_{\mathrm{sl}}^{b}$ in semileptonic decays of $b$ quarks to muons of "wrong charge", i.e., a muon of charge opposite to the sign of the charge of the original $b$ quark, induced through $B_{q}^{0} \leftrightarrow \bar{B}_{q}^{0}$ oscillations [5]:

$$
a_{\mathrm{sl}}^{b} \equiv \frac{\Gamma\left(\bar{B} \rightarrow B \rightarrow \mu^{+} X\right)-\Gamma\left(B \rightarrow \bar{B} \rightarrow \mu^{-} X\right)}{\Gamma\left(\bar{B} \rightarrow B \rightarrow \mu^{+} X\right)+\Gamma\left(B \rightarrow \bar{B} \rightarrow \mu^{-} X\right)}=A_{\mathrm{sl}}^{b} .
$$

The asymmetry $a_{\mathrm{sl}}^{b}$ can be measured from the inclusive muon charge asymmetry

$$
a \equiv \frac{n^{+}-n^{-}}{n^{+}+n^{-}}
$$

where $n^{+}$and $n^{-}$correspond to the number of positive and negative muons satisfying the kinematic selections. For the asymmetry $a$, the signal comes from $B_{q}^{0}$ mixing, followed by the semileptonic decay. In addition to the background already considered for $A$, the direct production of $c$ and light quark pairs followed by their semileptonic decays constitutes an additional source of muons contributing to $a$.

We use two data sets: the inclusive muon set, collected with single muon triggers, has $1.5 \times 10^{9}$ muons, and the like-sign dimuon set, collected with dimuon triggers, has $3.7 \times 10^{6}$ events. From these data sets we obtain, respectively, the single muon charge asymmetry $a=+0.00955 \pm 0.00003$ (stat), and the like-sign dimuon charge asymmetry $A=+0.00564 \pm 0.00053$ (stat). We correct these asymmetries for muons from kaon and pion decay, hadrons that punch through the calorimeter and iron toroid and get to the outer muon detector, and fake muon candidates, and also correct for the detector asymmetry. These corrections are measured with data with minimal use of simulation. By far, the largest correction is due to kaon decay. The corrected asymmetries are $a_{S}$ and $A_{S}$ respectively. Finally we obtain $A_{\mathrm{sl}}^{b}$ by dividing the corrected asymmetries by a dilution factor, taken form the Monte Carlo simulation, to account for non-direct semileptonic decays: $A_{\mathrm{sl}}^{b}=$ $a_{S} /(0.070 \pm 0.006)=+0.0094 \pm 0.0112$ (stat) \pm 0.0214 (syst), and $A_{\mathrm{sl}}^{b}=A_{S} /(0.486 \pm 0.032)=$ $-0.00736 \pm 0.00266$ (stat) \pm 0.00305 (syst). A third and more accurate measurement is obtained 


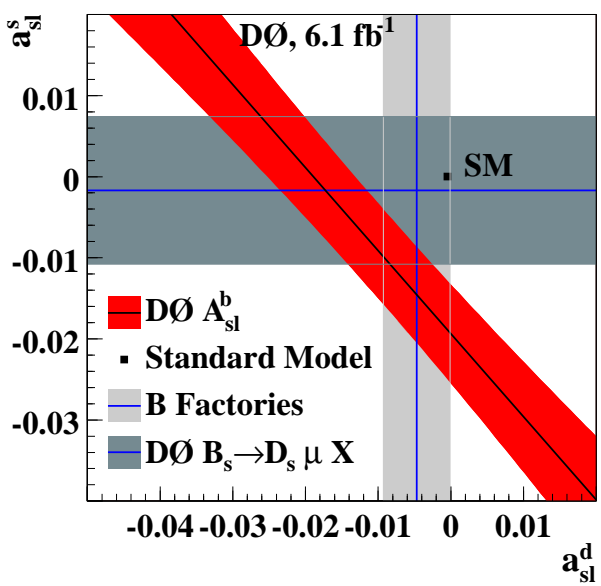

Figure 1: (Color online) Comparison of $A_{\mathrm{sl}}^{b}$ in data with the SM prediction for $a_{\mathrm{sl}}^{d}$ and $a_{\mathrm{sl}}^{s}$. Also shown are other measurements of $a_{\mathrm{sl}}^{d}=-0.0047 \pm 0.0046[16,17,18]$ and $a_{\mathrm{sl}}^{s}=-0.0017 \pm 0.0091$ [19]. The bands represent the \pm 1 standard deviation uncertainties on each measurement.

from the linear combination $A-\alpha a$ which cancels some correlated uncertainties. The total uncertainty of $A_{\mathrm{sl}}^{b}$ is minimized by setting $\alpha=0.959$. With this value of $\alpha$ we obtain our final result: $A_{\mathrm{sl}}^{b}=-0.00957 \pm 0.00251$ (stat) \pm 0.00146 (syst). It is consistent with our previous measurement [15] obtained with $1 \mathrm{fb}^{-1}$ and supersedes it. This result differs by 3.2 standard deviations from the standard model prediction for $A_{\mathrm{sl}}^{b}$ of Eq. 5. Consistency checks and a breakdown of uncertainties are described in detail in [1].

The measured value of $A_{\mathrm{sl}}^{b}$ places a constraint on the charge asymmetries in semileptonic decays of $B_{d}^{0}$ and $B_{s}^{0}$ mesons and on the $C P$-violating phases of the $B_{d}^{0}$ and $B_{s}^{0}$ mixing matrices, as given by Eqs. 3 and 4. Figure 1 presents our measurement of $A_{\mathrm{sl}}^{b}$ in the $a_{\mathrm{sl}}^{d}-a_{\mathrm{sl}}^{s}$ plane, together with direct measurements of $a_{\mathrm{sl}}^{d}$ from the $B$-factories [16, 17, 18] and of our independent measurement of $a_{\mathrm{sl}}^{s}$ in $B_{s}^{0} \rightarrow D_{s} \mu X$ decays [19]. Additional comparisons and combinations of these results with previous measurements sensitive to the same physics effect are given in Ref. [1].

\section{References}

[1] V.A. Abazov et al. (D0 Collaboration), Phys. Rev. D 82, 032001 (2010).

[2] V.A. Abazov et al. (D0 Collaboration), Phys. Rev. Lett. 105, 081801 (2010).

[3] M. Kobayashi and T. Maskawa, Prog. Theor. Phys. 49, 652 (1973).

[4] A. Lenz and U. Nierste, J. High Energy Phys. 0706, 072 (2007).

[5] Y. Grossman et al., Phys. Rev. Lett. 97, 151801 (2006).

[6] C. Amsler et al., Phys. Lett. B 667, 1 (2008), and 2009 partial update for the 2010 edition, and references therein.

[7] L. Randall and S. Su, Nucl. Phys. B 540, 37 (1999).

[8] J.L. Hewett, arXiv:hep-ph/9803370 (1998). 
[9] G.W.S. Hou, arXiv:0810.3396 [hep-ph] (2008).

[10] A. Soni et al., Phys. Lett. B 683, 302 (2010); A. Soni et al., arXiv:1002.0595 (2010) [hep-ph] and references therein.

[11] M. Blanke, A. J. Buras, A. Poschenrieder, C. Tarantino, S. Uhlig and A. Weiler, JHEP 0612, 003 (2006). W. Altmannshofer, A. J. Buras, S. Gori, P. Paradisi and D. M. Straub, Nucl. Phys. B 830, 17 (2010).

[12] A.D. Sakharov, Pisma Zh. Eksp. Teor. Fiz. 5, 32 (1967) [Sov. Phys. JETP Lett. 5, 24 (1967)].

[13] M.B. Gavela, P. Hernandez, J. Orloff, O. Pene, Mod. Phys. Lett. A 9, 795 (1994); M.B. Gavela, P. Hernandez, J. Orloff, O. Pene, C. Quimbay, Nucl. Phys. B 430, 382 (1994); P. Huet and E. Sather, Phys. Rev. D 51, 379 (1995).

[14] V.M. Abazov et al. (D0 Collaboration), Nucl. Instrum. Methods Phys. Res. A 565, 463 (2006).

[15] V.M. Abazov, et al. (D0 Collaboration), Phys. Rev. D 74, 092001 (2006).

[16] B. Aubert et al. (Babar Collaboration), Phys. Rev. Lett. 96, 251802 (2006); B. Aubert et al. (Babar Collaboration), arXiv:hep-ex/0607091 (2006).

[17] E. Nakano et al. (Belle Collaboration), Phys. Rev. D 73, 112002 (2006).

[18] E. Barberio et al. (HFAG), arXiv:0808.1297 [hep-ex] (2008).

[19] V.M. Abazov et al. (D0 Collaboration), arXiv:0904.3907 [hep-ex] (2009), accepted for publication in Phys. Rev. D. 